\title{
Productividad y calidad de los cuerpos fructíferos de los hongos comestibles Pleurotus pulmonarius RN2 y P. djamor RN81 y RN82 cultivados sobre sustratos lignocelulósicos
}

\author{
Aracelly Vega ${ }^{*}$, Heriberto Franco \\ Universidad Autónoma de Chiriquí, Centro de Investigación en Recursos Naturales, Apartado Postal 0427, \\ Chiriquí-Panamá (e-mail: aravega@cwpanama.net). \\ * Autor a quien debe dirigirse la correspondencia
}

Recibido Sep. 26, 2012; Aceptado Nov. 19, 2012; Versión final recibida Dic. 01, 2012

\begin{abstract}
Resumen
Parámetros tales como eficiencia biológica (EB), tasa de productividad (TP), tamaño de los cuerpos fructíferos, grasa, fibra, carbohidratos totales, valor de energía y proteínas totales, fueron determinados y analizados para hongos comestibles de la cepa importada Pleurotus pulmonarius RN2 y dos cepas nativas de Panamá P.djamor RN81 y RN82, cultivadas sobre paja de arroz (Oriza sativa L), rastrojo y tuza de maíz (Zea maíz L.). El objetivo fue establecer si existían diferencias significativas en estos parámetros, por efecto de la cepa, el tipo de sustrato o su interacción. Más del $50 \%$ de los hongos cosechados presentó un tamaño entre $5 \mathrm{y} 10 \mathrm{~cm}$. Se destaca un alto contenido de proteína para el hongo $P$. djamor RN82 crecido sobre tuza de maíz (43.07\%) y una alta eficiencia biológica para $P$. pulmonarius RN2 $(75.65 \%)$. Se obtuvo diferencias significativas para la eficiencia biológica, proteínas, carbohidratos y fibra por efecto de la interacción cepa sustrato.
\end{abstract}

\section{Productivity and quality of the fruiting bodies of edible mushrooms Pleurotus pulmonarius RN2 and P. djamor RN81 and RN82 grown on different lignocellulosic substrates}

\begin{abstract}
Parameters such as biological efficiency (BE), productivity rate (TP), size of fruiting bodies, fat, fiber, total carbohydrate, energy value and total protein, were determined and analyzed for edible mushrooms, Pleurotus pulmonarius RN2, a foreign strain and two native strains of Panama: P. djamor RN81 and RN82, all grown on rice straw (Oryza sativa L.), corn stubble and husk (Zea maize L.). The objective was to establish if there were significant statistical differences in these parameters due to strain, type of substrate or their interaction. Over $50 \%$ of the harvested mushrooms showed sizes between 5 and $10 \mathrm{~cm}$. Outstanding results were the high protein content for fungus $P$. djamor RN82 grown on corn husk (43.07\%) and high biological efficiency for $P$. pulmonarius RN2 (75.65\%). Significant differences were obtained for biological efficiency, proteins, carbohydrates and fiber due to the strain-substrate interaction.
\end{abstract}

Keywords: biological efficiency, protein, pleurotus pulmonarius, rice straw, corn stubble 


\section{INTRODUCCIÓN}

Los hongos tienen la capacidad de crecer sobre una gran variedad de sustratos. La bioconversión de residuos agrícolas e industriales a alimentos, ha atraído la atención del mundo en años recientes, siendo el cultivo de hongos un método eficiente para la disposición de los residuos agrícolas, como también la producción de alimentos nutritivos (Ingale y Ramteke, 2010). La elección del sustrato depende de su disponibilidad, costo y de su contenido de nutrientes; los hongos utilizan $\mathrm{C}, \mathrm{N}$ y otros nutrientes y metabolitos, que contienen los residuos sólidos para su propio metabolismo y que requieren para su óptimo crecimiento y fructificación (Nyochembeng et al., 2008). Las especies de Pleurotus crecen rápido y exitosamente, bajo condiciones semi controladas en pequeños espacios y tienen la habilidad de utilizar varios sustratos lignocelulósicos (Bhatti et al., 2007), lo cual hace posible su cultivo en diferentes partes del mundo, siendo el segundo género de hongos más cultivado en términos de la producción mundial. La habilidad de los hongos de crecer en una amplia variedad de residuos lignocelulósicos, es atribuída a su capacidad de secretar enzimas degradadoras como celulasas, hemicelulasas, xilanasas, LiP, MnP y lacasa, que se ha demostrado que varían en especies de Pleurotus spp., de acuerdo a la cepa y tipo de sustrato, durante sus distintos estados de desarrollo (Periasamy y Natarajan, 2004).

Algunas de las especies de Pleurotus spp. cultivadas son: el hongo ostra árbol ( $P$. ostreatus), ostra gris $(P$. sajor-caju), ostra blanco ( $P$. florida), el ostra oro ( $P$. citrinopileatus), ostra rosado ( $P$. flabellatus) y el hongo ostra negro (P. sapidus) (Martínez-Carrera, 1998; Frimpong-Manso et al., 2011).Las distintas especies de hongos tipo ostras, han sido cultivadas en una diversidad de sustratos y combinaciones de ellos, como: paja de arroz y paja de trigo, paja de frijol de soya, paja de cebada, residuos de maíz, residuos de césped, obteniendo una alta eficiencia biológica (peso del hongo fresco/ peso seco del sustrato x 100) entre 72 y $208 \%$ (Zhang et al., 2002, Ahmed et al., 2009, Jafarpour et al., 2011). Se ha reportado que la paja de trigo y paja de arroz, son los mejores sustratos para el cultivo de diversas especies de Pleurotus, en función de su composición química, la cual provee una reserva de celulosa, hemicelulosas y lignina, que es utilizada por el hongo durante el crecimiento del micelio y durante la fructificación (Yildiz et al, 2002).

Los hongos son considerados un alimento completo, saludable y apto para personas de todas las edades, puesto que son ricos en proteína, fibra dietética (principalmente polisacáridos digeribles y quitina), vitaminas (especialmente C y B), y minerales (K, Na, $\mathrm{P}, \mathrm{Zn}, \mathrm{Mg}$ ); el $80-90 \%$ de su peso es agua y de $8-10 \%$ es fibra (Singh et al., 2011) y bajos en grasa total, con una alta proporción (72-85\%) de ácidos grasos poliinsaturados en relación al contenido total de grasas (Dundar et al., 2008). Para $P$. ostreatus y P. sajorcaju cultivados sobre paja de arroz, se han encontrado valores de grasa total, ceniza, fibra total y proteína total entre $4.99-6.32 \%, 5.59-6.13 \%, 9.60-9.86 \%$ y $13.0-13.1 \%$, respectivamente (Bonatti et al., 2004).

Cada año se genera una gran cantidad de residuos, provenientes de las cosechas de productos utilizados en la alimentación humana, como el arroz, maíz, trigo, café, frijol de soya, azúcar y algodón, que se estima en 3736.05 millones de toneladas métricas (TM); de las cuales 698.10 millones de TM, corresponden a residuos de la cosecha de arroz y 1729.92 millones de TM, a los residuos de la cosecha del maíz (Zhang et al., 2012). Todos estos residuos, representan una materia prima apta para la producción de hongos comestibles. En esta investigación se evaluaron la productividad y de calidad nutricional, de los hongos de una cepa importada de $P$. pulmonarius RN2 y dos cepas nativas de Panamá de $P$. djamor RN81 y RN82 por efecto de su cultivo sobre residuos agrícolas disponibles en Panamá como la paja de arroz (Oriza sativa L.), rastrojo y tuza de maíz (Zea maíz L.).

\section{METODOLOGÍA}

Se utilizaron dos cepas nativas: RN81 y la RN82, de la especie Pleurotus djamor, que fueron colectadas en la Granja del Centro Pastoral Santa Fé, Darién, República de Panamá, en septiembre del 2002 y la cepa RN2, de la especie Pleurotus pulmonarius, procedente de USA, recibida en marzo de 1998. Estas tres cepas se mantienen en el cepario del Centro de Investigación en Recursos Naturales, de la Universidad Autónoma de Chiriquí, Panamá, en una incubadora a $25 \pm 1$ _C. Se mantienen en medio papa dextrosa agar (EMD Chemicals Inc.), con transferencia periódica mensual.

\section{Preparación del sustrato, siembra y cosecha}

La paja de arroz (PA) y el rastrojo de maíz (RM) fueron picados hasta un tamaño de partícula de $3 \mathrm{~cm}$ aproximadamente y la tuza de maíz (TM) fue molida en un molino de cuchillas giratorias hasta un tamaño de partícula de $2 \mathrm{~cm}$. Con cada sustrato se llenan unas canastas de malla de alambre y se sumergen en agua caliente a $85^{\circ} \mathrm{C}$ durante una hora. Se drena y se coloca en un cuarto libre de contaminación hasta que el sustrato alcance la temperatura ambiente (aproximadamente $25^{\circ} \mathrm{C}$ ). Las canastas se dejaron drenando toda la noche. La humedad que retuvo la paja de arroz fue de $73.4 \%$, rastrojo de maíz, $74.9 \%$ y la tuza de maíz, $62.2 \%$. Se colocó $1 \mathrm{~kg}$, en base húmeda, de sustrato en bolsas de polietileno de2 $\mathrm{kg}$ y se adicionaron $60 \mathrm{~g}$ 
de inóculo de las cepas de hongos crecidos sobre granos de sorgo (semilla), para un porcentaje de inoculación del $6 \%$ en peso. Por cada cepa se inocularon 15 bolsas, que después de la siembra fueron colocadas en el cuarto de incubación a una temperatura de $24^{\circ} \mathrm{C}$ y en oscuridad, por 15 días, tiempo en el cual el micelio cubrió completamente el sustrato. Después del tiempo de incubación, las bolsas fueron trasladadas al área de cosecha, en nuestro caso un invernadero con un sistema de control ambiental, para regular la temperatura a $24+2^{\circ} \mathrm{C}, 80-95 \%$ de humedad y una leve luminosidad. Bajo estas condiciones, las bolsas se mantienen en producción durante 30 días, dentro de los cuales se realizan las cosechas.

\section{Parámetros de productividad de Pleurotus spp.}

Para conocer el potencial de producción de las cepas estudiadas se determinó la eficiencia biológica (EB) y la tasa de producción. i) la eficiencia biológica $(E B \%)$ se determina expresando en porcentaje la relación entre el peso fresco de los hongos producidos y el peso del sustrato seco [EB (\%) $=$ (peso de hongos frescos/ peso del sustrato seco ${ }^{\star} 100 \%$ ]; y ii) la tasa de producción (TP) se determinó mediante la relación del porcentaje de eficiencia biológica entre el número total de días del proceso [TP=EB (\%)/número de días del proceso].

\section{Tamaño de los cuerpos fructíferos}

Para determinar el tamaño de los cuerpos fructíferos, se procedió a cosechar individualmente cada bolsa. Los hongos fueron medidos y clasificados de acuerdo al siguiente formato: A: hongos menores de $5 \mathrm{~cm}$, B: hongos entre $5-10 \mathrm{~cm}$ y hongos $\mathrm{C}$ : mayores a $10 \mathrm{~cm}$. El total cosechado de los cuerpos fructíferos por bolsa, fue clasificado por tamaño y pesado.

\section{Análisis nutricional}

Los hongos de cada una de las cepas, fueron cosechados de las bolsas, secados a $65^{\circ} \mathrm{C}$, molidos y tamizados a 20 "mesh"; los hongos tamizados de las 3 cosechas fueron mezclados para obtener una muestra compuesta, de cada combinación cepa sustrato, utilizando el proceso del cuarteo. Estas muestras se guardaron en bolsas "ziploc" y se mantuvieron a $10^{\circ} \mathrm{C}$, hasta someterlas a análisis utilizando la metodología oficial (AOAC, 2005). Los parámetros analizados fueron: proteína cruda, grasa cruda, fibra cruda. Cada muestra se analizó por triplicado y utilizando un gramo de muestra en cada caso. Para la determinación de proteína se utilizó el método Kjeldahl, utilizando digestor (digestion system 2000) y destilador (Kjeltec system 1002 Distilling Unit Tecator) de operación automática (método AOAC, 2005). La grasa cruda fue determinada por extracción con éter de petróleo usando un equipo Soxhlet (método AOAC, 2005). La fibra cruda fue analizada por digestión ácido y base usando un digestor modelo 30001 (método AOAC, 2005).

\section{Análisis estadístico}

Todos los resultados fueron analizados mediante prueba ANOVA y Tukey, para determinar la significancia estadística entre las distintas cepas, sustratos y su interacción, utilizando el programa estadístico STATGRAPHIC Centurion XVI.I

\section{RESULTADOS Y DISCUSIÓN}

El período total de cultivo de los hongos se extendió por 54 días, en los cuales se realizaron tres cosechas. En la tabla 1 se presentan los valores de los parámetros de productividad de los hongos. Los valores EB y tasa de productividad (TP), más alto fueron obtenidos para $P$. pulmonarius RN2 cultivado sobre paja de arroz, siendo de $75.65 \%$ y 1.44 , respectivamente. Los valores de EB y TP más bajos, se obtuvieron para $P$. djamor RN82 cultivado sobre tuza de maíz (15.94\% y 0.30 , respectivamente). No se encontraron diferencias estadísticas significativas $(P<0.05)$ para el factor cepas. Las diferencias estadísticas significativas se obtuvieron cuando se compara la EB de las cepas crecidas en distintos sustratos. Para la cepa $P$. pulmonarius RN2, la eficiencia biológica en función del sustrato siguió el orden paja de arroz/rastrojo de maíz/tuza de maíz (Fig. 1). Las EB y TP más bajas se obtuvieron para los hongos que crecieron sobre tuza de maíz. Las cepas nativas $P$. djamor RN81 y RN82, no presentaron diferencias estadísticas significativas cuando se cultivaron sobre paja de arroz y rastrojo de maíz. La EB de $P$. pulmonarius RN2 crecida sobre paja de arroz es similar a la reportada para el cultivo de $P$. sajor-caju $(75.5 \%$ EB) sobre paja de arroz cortada y con un tamaño de partícula de $2.5 \mathrm{~cm}$, pero con un porcentaje de inoculación en peso de $12 \%$ (Zhang et al., 2002).

Para la EB biológica de las especies $P$. sajor-caju, $P$. florida y $P$. eous, cultivadas sobra paja de frijol de soya cortada (1-2 cm de largo) y con una inoculación del $2 \%$, se han reportado valores de $60.72 \%, 56.64 \%$ y $76.76 \%$, respectivamente (Ingale y Ramteke, 2011). Para P. djamor IE-121e IE 218 cultivadas sobre paja de trigo, se ha reportado EB de $40.4 \%$ y $30.0 \%$ (Salmones et al., 2005), respectivamente, más altas que las 
encontrados en este trabajo para $P$. djamor RN82 (15.94-23.94\%) y más bajas que las encontradas para $P$. djamor RN81 (53.16-62.19\%). Se puede observar una clara tendencia de la cepa $P$. pulmonarius RN2 de ser más eficaz en la utilización del sustrato y producción de cuerpos fructíferos, bajo las condiciones ambientales controladas. Es posible que las cepas nativas $P$. djamor RN81 y RN82, necesiten condiciones de una mayor temperatura que $P$. pulmonarius RN2 (cepa importada), ya que son cepas adaptadas a las condiciones del trópico.

La diferencia en los parámetros de productividad de las distintas cepas sobre tuza de maíz en comparación con paja de arroz y rastrojo de maíz, puede ser explicado por la diferencia en la estructura de estos sustratos lignocelulósicos; la paja de arroz y el rastrojo de maíz al ser colonizados por el micelio del hongo, formaron una torta compacta, mientras que la tuza de maíz, a pesar de ser bien colonizada por el hongo, no forma una estructura compacta y al ser sometida a riego en el área de cosecha, se observó que el sustrato se disgregaba, perdiéndose algunas partículas de sustrato, lo cual pudo influir en una baja producción. En términos de composición química, la tuza de maíz, presentó una composición de lignina y celulosa similar a la paja de arroz y rastrojo de maíz (datos no mostrados), y una diferencia significativa en el contenido de hemicelulosas, siendo de $43 \%$ para la tuza de maíz y de $29 \%$ para la paja de arroz y rastrojo de maíz. En relación al tamaño de los cuerpos fructíferos, la mayor cantidad de ellos (sobre 50\%), fueron clasificados en el rango de $5-10 \mathrm{~cm}$ y no se encontraron diferencias significativas para este parámetro por efecto de la cepa, el sustrato o su interacción. El tamaño de los cuerpos fructíferos de los hongos $P$. dajmor y $P$. pulmonarius es acorde a los valores reportados para el género Pleurotus, que van de $5-20 \mathrm{~cm}$ en diámetro (Martínez-Carrera, 1998).

Tabla 1: Parámetros de productividad y tamaño de los cuerpos fructíferos de $P$. pulmonarius RN2 y $P$. djamor RN81 y RN82 cultivados sobre paja de arroz (PA), rastrojo de maíz (RM) y tuza (TM).

\begin{tabular}{|c|c|c|c|c|c|c|}
\hline \multirow{2}{*}{$\begin{array}{c}\text { Sustrato- } \\
\text { cepa }\end{array}$} & EB (\%) & T.P. & $\begin{array}{c}\text { Masa hongo } \\
\text { fresco }(\mathrm{g})\end{array}$ & & \multicolumn{3}{|c|}{ Tamaño del hongo } \\
\cline { 5 - 7 } & & & & $(\%) \mathrm{T} 1: 5 \mathrm{~cm}$ & $(\%) \mathrm{T} 2: 5-10 \mathrm{~cm}$ & $(\%) \mathrm{T}:>10 \mathrm{~cm}$ \\
\hline PA-RN2 & $75.65 \pm 5.27$ & $1.44 \pm 0.03$ & $3018 \pm 36$ & $34.40 \pm 1.66$ & $57.92 \pm 0.96$ & $11.51 \pm 2.73$ \\
\hline RM-RN2 & $62.22 \pm 4.53$ & $1.19 \pm 0.14$ & $1117 \pm 17$ & $16.59 \pm 7.71$ & $58.61 \pm 7.28$ & $24.81 \pm 8.42$ \\
\hline TM-RN2 & $69.31 \pm 4.48$ & $1.33 \pm 0.15$ & $2103 \pm 31$ & $14.07 \pm 4.07$ & $55.74 \pm 10.50$ & $30.20 \pm 13.95$ \\
\hline PA-RN81 & $55.79 \pm 2.27$ & $1.03 \pm 0.04$ & $2482 \pm 37$ & $38.22 \pm 6.09$ & $56.32 \pm 4.13$ & $5.26 \pm 2.81$ \\
\hline RM-RN81 & $53.16 \pm 8.07$ & $0.98 \pm 0.15$ & $1279 \pm 19$ & $15.59 \pm 1.01$ & $57.39 \pm 5.45$ & $27.03 \pm 1.92$ \\
\hline TM-RN81 & $62.90 \pm 4.78$ & $1.16 \pm 0.09$ & $2003 \pm 42$ & $16.15 \pm 0.89$ & $57.53 \pm 6.22$ & $26.32 \pm 5.87$ \\
\hline PA-RN82 & $19.69 \pm 0.83$ & $0.36 \pm 0.02$ & $2765 \pm 33$ & $20.36 \pm 6.24$ & $66.06 \pm 10.68$ & $3.91 \pm 1.44$ \\
\hline RM-RN82 & $23.14 \pm 1.71$ & $0.40 \pm 0.04$ & $904 \pm 19$ & $8.42 \pm 2.40$ & $35.40 \pm 6.55$ & $56.18 \pm 8.85$ \\
\hline TM-RN82 & $15.94 \pm 3.16$ & $0.30 \pm 0.06$ & $2379 \pm 28$ & $18.31 \pm 7.13$ & $55.43 \pm 7.22$ & $33.45 \pm 9.81$ \\
\hline
\end{tabular}

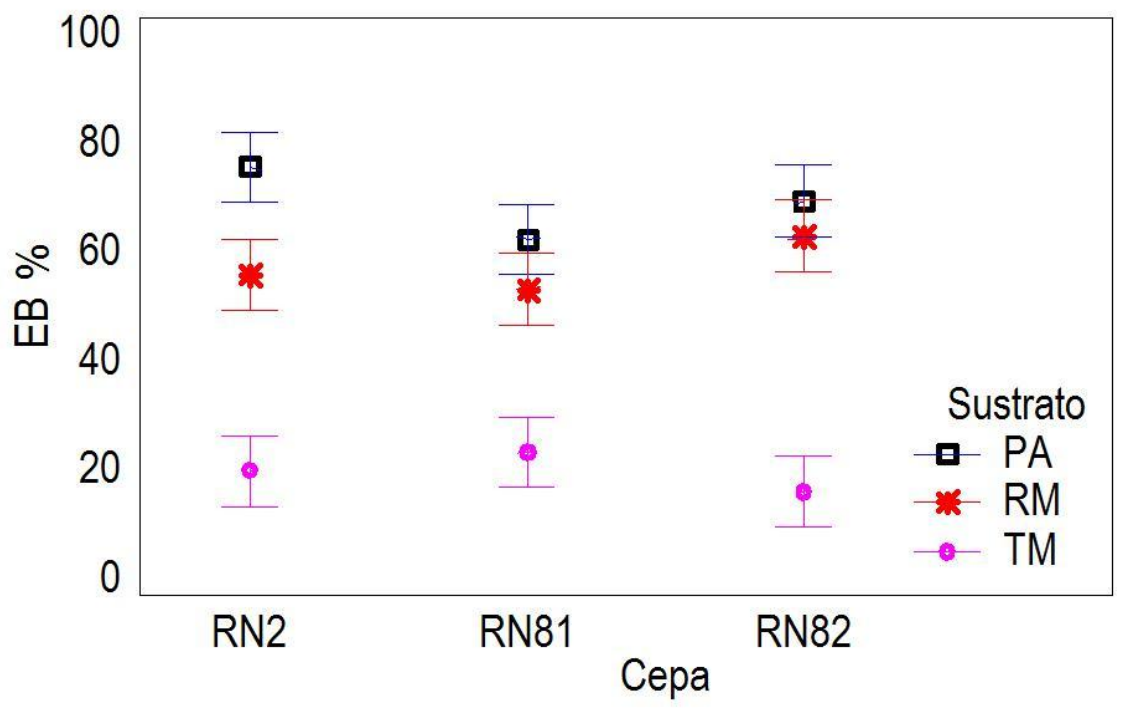

Fig. 1: Prueba Tukey de significancia estadística al 95\% de intervalo de confianza para la eficiencia biológica (EB) de P. pulmonarius (RN2) y P. djamor (RN81 y RN82) cultivados sobre paja de arroz (PA), rastrojo de maíz (RM) y tuza de maíz (TM) 
En la tabla 2 se presentan los resultados de la composición química de los cuerpos fructíferos de las distintas combinaciones cepa-sustrato. Para la fibra cruda, se observa una diferencia significativa entre la cepa $P$. pulmonarius RN2 y las cepas de $P$. djamor RN 82 y RN81 (figura 2). El valor más alto de fibra cruda fue obtenido para los hongos de la cepa $P$. djamor RN81 cultivados sobre tuza de maíz que fue de $12.15 \%$. Los valores de fibra cruda para las dos cepas nativas están en el rango de $8-10 \%$, indistintamente del sustrato en el que se cultivaron y en los hongos de la cepa $P$. pulmonarius aproximadamente en $4 \%$, en los tres sustratos evaluados. Entonces, se puede señalar que el contenido de fibra cruda es un componente nutricional que diferencia a las cepas de $P$. djamor nativas de la $P$. pulmonarius importada. Los valores de fibra cruda para $P$. djamor RN81 y RN82 son similares a los reportados para setas de $P$. ostreatus cosechados sobre paja de arroz (Jonathan et al., 2012), que fueron entre 8.5 y $10.16 \%$ y para $P$. sajor-caju, $P$. florida y $P$. eous, crecidos sobre paja de frijol de soya, que estuvo en el rango de 10.9 a $12 \%$ (Ingale y Ramteke, 2010).
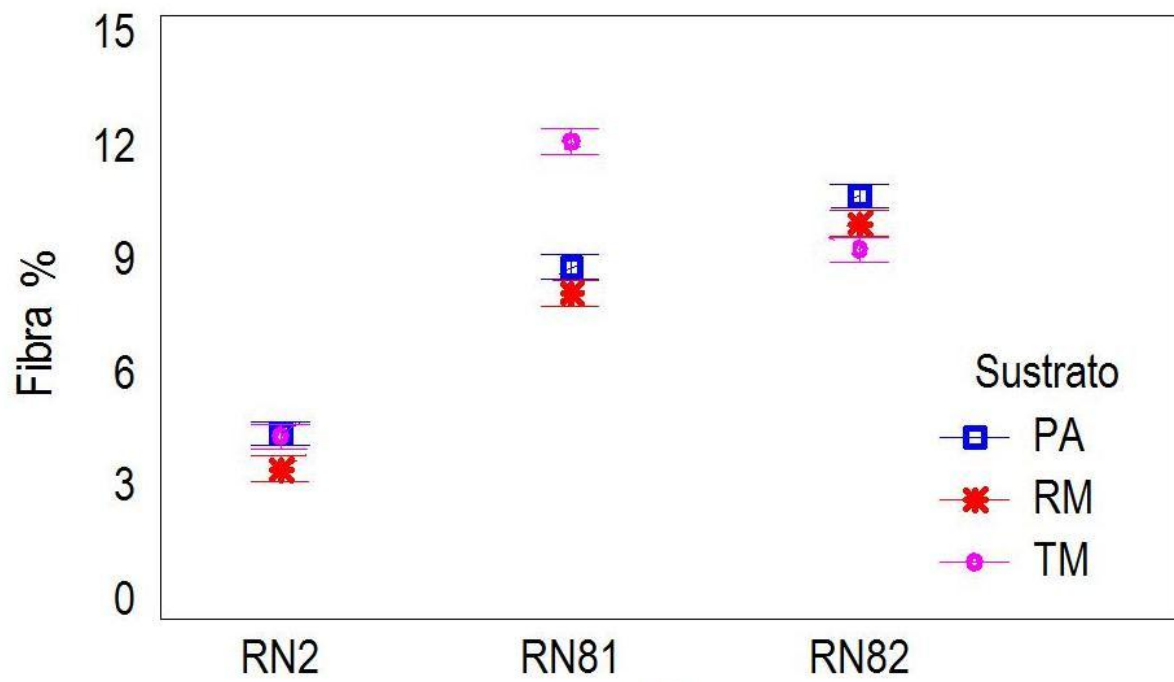

RN81

Cepa

Fig. 2: Prueba Tukey de significancia estadística al $95 \%$ de intervalo de confianza para el contenido de fibra cruda (\%) de $P$. pulmonarius (RN2) y $P$. djamor (RN81 y RN82) cultivados sobre paja de arroz (PA), rastrojo de maíz $(R M)$ y tuza de maíz (TM).

Tabla 2: Composición química de los hongos P. pulmonarius RN2 y P. djamor RN81 y RN82

\begin{tabular}{|c|c|c|c|c|c|}
\hline Sustrato-cepa & Grasa (\%) & Fibra (\%) & $\begin{array}{c}\text { Proteína } \\
(\%)\end{array}$ & $\begin{array}{c}\text { Carbohidratos } \\
\text { totales }(\%)\end{array}$ & $\begin{array}{c}\text { Valor de energía } \\
(\mathrm{kcal} / 100 \mathrm{~g})\end{array}$ \\
\hline PA-RN2 & $2.85 \pm 0.36$ & $4.44 \pm 0.26$ & $28.24 \pm 0.41$ & $49.23 \pm 0.13$ & $335.53 \pm 4.21$ \\
\hline RM-RN2 & $3.04 \pm 0.28$ & $3.48 \pm 0.08$ & $23.54 \pm 0.26$ & $52.44 \pm 0.46$ & $331.32 \pm 1.39$ \\
\hline TM-RN2 & $3.05 \pm 0.13$ & $4.34 \pm 0.08$ & $32.82 \pm 0.65$ & $44.28 \pm 0.66$ & $335.84 \pm 1.19$ \\
\hline PA-RN81 & $1.22 \pm 0.51$ & $8.82 \pm 0.21$ & $32.93 \pm 0.14$ & $40.53 \pm 0.64$ & $304.86 \pm 2.92$ \\
\hline RM-RN81 & $1.57 \pm 0.25$ & $8.12 \pm 0.15$ & $37.36 \pm 0.44$ & $34.42 \pm 0.27$ & $301.27 \pm 1.18$ \\
\hline TM-RN81 & $2.19 \pm 0.01$ & $12.15 \pm 0.21$ & $33.87 \pm 0.19$ & $33.67 \pm 0.27$ & $289.83 \pm 1.16$ \\
\hline PA-RN82 & $2.05 \pm 0.33$ & $10.70 \pm 0.20$ & $41.61 \pm 0.09$ & $29.88 \pm 0.73$ & $304.38 \pm 0.55$ \\
\hline RM-RN82 & $0.21 \pm 0.1$ & $9.99 \pm 0.29$ & $35.78 \pm 0.33$ & $35.38 \pm 0.77$ & $290.92 \pm 3.58$ \\
\hline TM-RN82 & $1.98 \pm 0.48$ & $9.30 \pm 0.40$ & $43.07 \pm 0.19$ & $27.39 \pm 0.54$ & $299.65 \pm 2.22$ \\
\hline
\end{tabular}

Con relación al contenido de grasa, se obtuvieron bajos valores para todas las combinaciones cepas sustratos, aunque los hongos $P$. pulmonarius RN2 crecidos sobre paja de arroz presentaron los valores más altos de grasa (3\%). Para un mismo sustrato, no se observaron diferencias significativas en el contenido de grasa de los hongos de las tres cepas en estudio. El bajo contenido de grasas es una cualidad nutricional por la que destacan los hongos. Además, se ha informado contenidos de lípidos de 4.3 y $4.2 \%$, en hongos $P$. ostreatus y $P$. cornucopiae, respectivamente, crecidos sobre cáscaras de semilla de algodón, con una alta proporción de ácidos grasos poliinsaturados, que producen efectos beneficiosos en la salud y su concentración y grado de insaturación, dependen de los componentes nutricionales, temperatura y oxígeno, bajo los que crecen los hongos (Pedneault et al., 2007). 
El contenido de proteína cruda en los hongos de las cepas nativas $P$. djamor RN81 y RN82 es significativamente mayor que el de la cepa importada $P$. pulmonarius RN2, para todos los sustratos (Fig. 3 ). Para los hongos de la cepa $P$. pulmonarius RN2 y $P$. djamor RN82, el contenido de proteína cruda fue significativamente mayor en paja de arroz y tuza de maíz, en relación con los hongos de la cepa $P$. dj amor RN81, que sólo presentó un contenido de proteína mayor cuando fue cultivada sobre rastrojo de maíz. Los resultados demuestran una alta variabilidad en el contenido de proteína cruda (23-43\%), dependiendo del sustrato en donde se cultiva cada cepa. Estos resultados confirman lo expresado en la literatura, con relación al contenido de proteína en hongos del género Pleurotus, en donde el contenido de proteína varía considerablemente entre y dentro de especies, entre 10 y 40\% (Chang, 2004, Ingale y Ramteke, 2010). Para $P$. ostreatus, crecido sobre paja de arroz, se ha informado un contenido de proteína de $27.42 \%$ (Jonathan et al., 2012), que es similar al obtenido para $P$. pulmonarius RN2, pero inferior a los obtenido para las dos cepas de $P$. djamor RN81 y RN82.

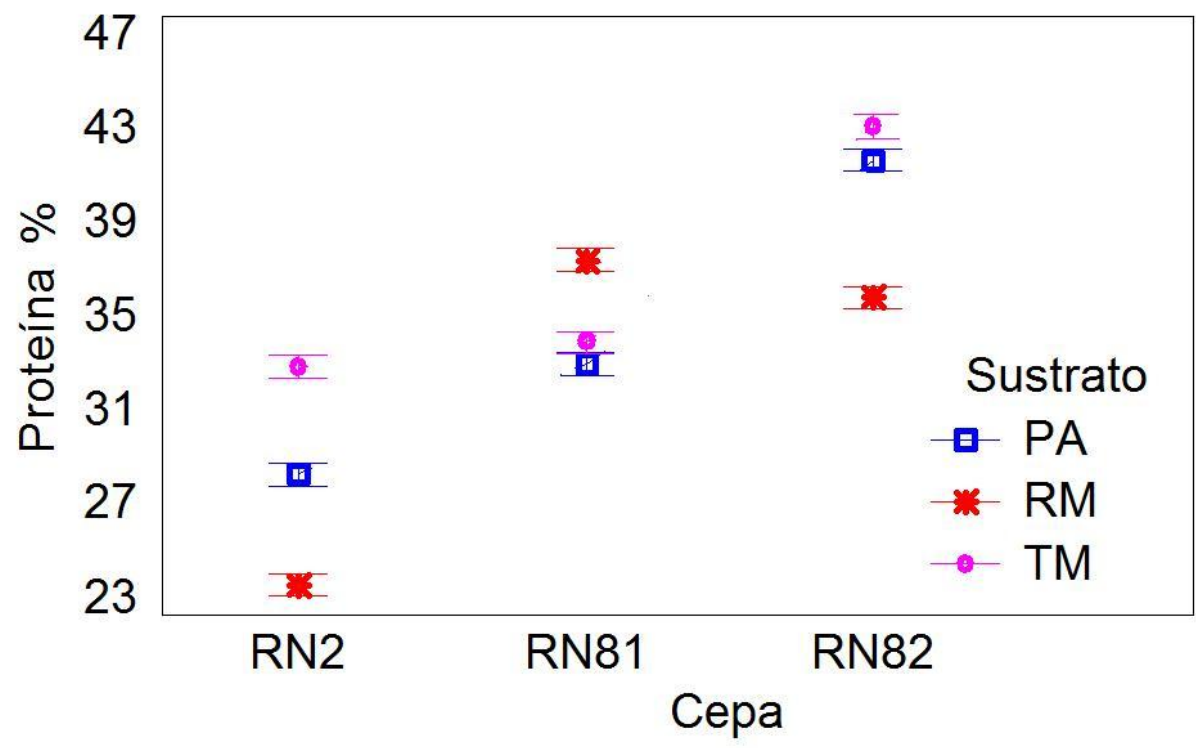

Fig. 3: Prueba Tukey de significancia estadística al 95\% de intervalo de confianza, para el contenido de proteína cruda (\%) de P. pulmonarius (RN2) y P. djamor (RN81 y RN82) cultivados sobre paja de arroz (PA), rastrojo de maíz (RM) y tuza de maíz (TM).

Los carbohidratos totales, determinados por diferencia, presentaron valores entre 52.44 y $27.39 \%$ (Tabla 2). Existen diferencias significativas (Fig. 4) en el contenido de carbohidratos totales en los hongos de $P$. pulmonarius RN2 (mayor en todos los sustratos) y Pleurotus djamor RN81 y RN82. El contenido de carbohidratos totales en los hongos comestibles siguió el orden RN2> RN81> RN82. Estos valores se encuentran en el rango de los reportados para $P$. sajor-caju, crecido sobre paja de trigo $(52.34 \%), P$. ostreatus, crecidos sobre residuos de la cosecha del arroz (49.9\%), P. eryngii (39.85\%), P. ostreatus (37.87\%) y P. sajor-caju (29.68\%), P. florida (32.53\%) y P. ostreatus $(32.33 \%)$, crecidos sobre paja de trigo (Dunder et al., 2008; Dunkwal y Jood, 2009; Michael et al., 2011).

La variación en el contenido de carbohidratos totales en los hongos de las cepas de Pleurotus spp., puede ser explicado porque el contenido de carbohidratos en cada cepa demuestra especificidad topológica en el cuerpo fructífero y difiere significa-tivamente entre las especies de Pleurotus, como también dentro de una misma especie, como se ha reportado para la variación en el contenido de glucano en los cuerpos fructíferos de Pleurotus ostreatus (Synytsya et al., 2008). En este estudio el contenido de carbohidratos totales más altos se obtiene para $P$. djamor RN82, crecido sobre rastrojo de maíz y el más bajo para $P$. djamor RN82, crecido sobre tuza de maíz.

La energía de los cuerpos fructíferos de Pleurotus spp. presenta valores entre 290 y $335 \mathrm{kcal} / 100 \mathrm{~g}$ de hongo en base seca, que son superiores a los informados para $P$. eryngii, $P$. ostreatus y $P$. sajor-caju, cultivados sobre tallos de trigo, que fueron de $276.33,243.66$ y $229.42 \mathrm{kcal} / 100 \mathrm{~g}$ de hongo en base seca (Dundar et al., 2008) e inferiores al reportado para $P$. sajor-caju, cultivado sobre paja de trigo, que fue de $412 \mathrm{kcal} / 100 \mathrm{~g}$ de hongo en base seca (Dunkwal y Jood, 2009). Los resultados obtenidos son similares a los reportados para $P$. djamor (ostra rosado) y $P$. pulmonarius, que fueron de 356 y $355 \mathrm{kcal} / 100 \mathrm{~g} \mathrm{b.s.,}$ 
respectivamente (Stamet, 2005).Los cuerpos fructíferos de los hongos $P$. pulmonarius RN2 presentaron valores de energía mayores y con diferencias significativas en relación a los valores de energía para $P$. djamor RN81 y RN82 (Fig. 5).

En la tabla 3 se presenta un análisis comparativo del contenido de proteína, grasa y valores de energía de productos de consumo diario y de los cuerpos fructíferos de $P$. pulmonarius RN2 y $P$. djamor RN82, crecidos sobre tuza de maíz y que presentaron altos valores de proteína. Se observa que los contenidos de proteína en los hongos son superiores a los productos cárnicos, similares o superiores al contenido de proteína en el frijol de soya. En cuanto al valor energético, los hongos contienen más calorías que las carnes y menos que el frijol de soya. Estos resultados confirman el alto valor nutricional de los hongos comestibles.

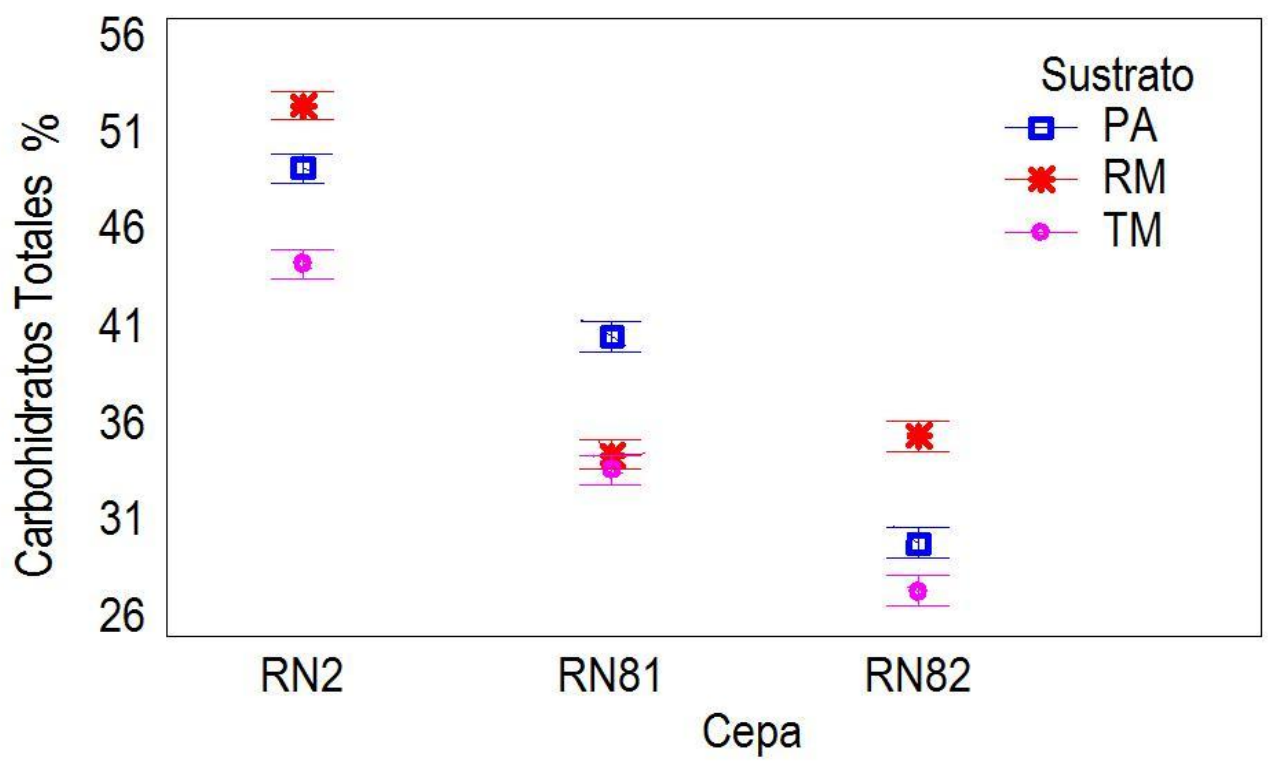

Fig. 4: Prueba Tukey de significancia estadística al 95\% de intervalo de confianza, para el contenido de carbohidratos totales (\%) de $P$. pulmonarius (RN2) y $P$. djamor (RN81 y RN82) cultivados sobre paja de arroz (PA), rastrojo de maíz (RM) y tuza de maíz (TM).

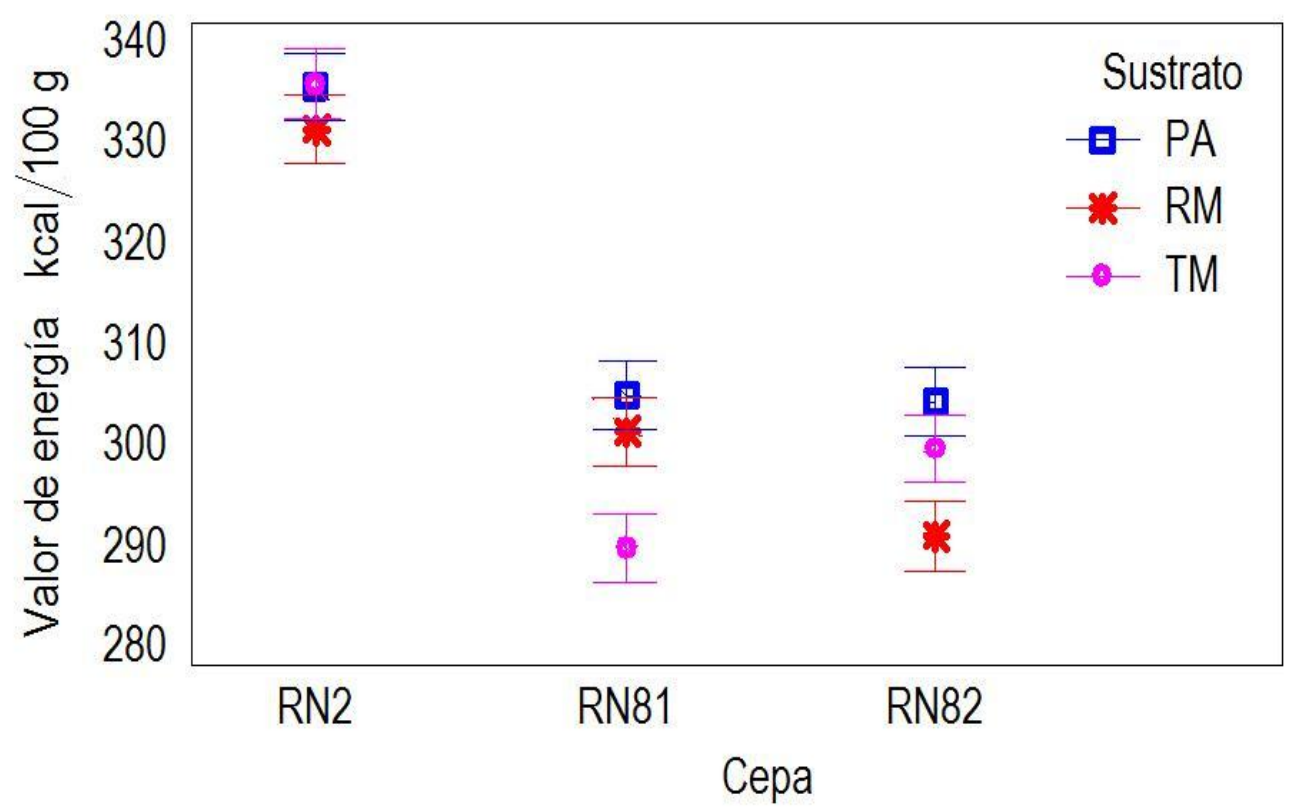

Fig. 5: Prueba Tukey de significancia estadística al 95\% de intervalo de confianza, para los valores de energía (kcal/ $100 \mathrm{~g}$ de hongo en base seca) de $P$. pulmonarius (RN2) y P. djamor (RN81 y RN82) cultivados sobre paja de arroz (PA), rastrojo de maíz (RM) y tuza de maíz (TM). 
Tabla 3: Análisis comparativo del contenido de energía, proteína y grasa en alimentos de consumo masivo y cuerpos fructíferos de hongos del género Pleurotus spp. cultivados sobre diferentes sustratos.

\begin{tabular}{|c|c|c|c|}
\hline Alimento & Energía kcal/100 g b.s. & Proteína (\%) & Grasa (\%) \\
\hline P. pulmonarius $\mathrm{RN} 2$, cultivado sobre tuza de maíz & 335.84 & 32.82 & 3.05 \\
\hline P. djamor RN82, cultivado sobre tuza de maíz & 299.65 & 43.07 & 1.98 \\
\hline${ }^{\star}$ Pechuga de pollo sin piel cocida (*INCAP, 2007) & 151 & 28.98 & 3.03 \\
\hline${ }^{*}$ Carne semimagra de cerdo asada & 260 & 27.63 & 15.76 \\
\hline${ }^{*}$ Carne de res semimagra asada & 291 & 26.42 & 19.71 \\
\hline${ }^{*}$ Frijol de soya, grano seco & 416 & 36.49 & 19.94 \\
\hline
\end{tabular}

\section{CONCLUSIONES}

Se encontraron diferentes valores para la productividad del cultivo de la cepa importada $P$. pulmonarius RN2 y las cepas nativas $P$. djamor RN81 y RN82, en función del tipo de sustrato lignocelulósico en el que se cultivaron. En relación a la eficiencia biológica y la tasa de productividad, en términos generales, se obtuvo bajos valores para los hongos cultivados sobre tuza de maíz y altos valores para los cultivados sobre paja de arroz. Sin embargo, el contenido nutricional de los cuerpos fructíferos presentó valores de proteína y fibra más altos para $P$. djamor RN81 y RN82, mientras que el contenido de carbohidratos, grasa y valores de energía fueron más altos para los hongos $P$. pulmonarius RN 2. Estos resultados demuestran que pueden obtenerse altos valores de productividad (eficiencia biológica y tasa de productividad),relacionada con la interacción de factores como la composición química, estructura morfológica del sustrato y condiciones ambientales, que favorece el crecimiento de micelio y la fructificación, para determinada interacción cepasustrato, pero el contenido nutricional en relación a determinados parámetros nutricionales, como el contenido de proteína y fibra, pueden ser mayor en aquellas cepas que han tenido valores más bajos en los parámetros de productividad.

\section{REFERENCIAS}

Bhatti, M.I. y otros autores, Growth, development and yield of oyster mushroom, Pleurotus ostreatus (Jacq.Ex. Fr) Kummer as affected by different spawn rates, Pak. J. Bot. 39(7), 2685-2692 (2007).

Bonatti, M., P. Karnopp, H.M. Soaresn y S.A. Furlan, Evaluation of Pleurotus ostreatus and Pleurotus sajorcaju nutritional charactetistics when cultivated in different lignocellulosic wastes, Food Chemistry 88, 425428 (2004).

Chang, S.T., Mushrooms: cultivation, nutritional value, medicinal effect, and environmental impact, S.T. Chang y P.G. Miles (ed.), pp 477, CRC Press LLC, Boca Ratón, Florida, USA (2004).

Dundar, A., H. Acay, y A. Yildiz, Yield performances and nutricional contents of three oyster mushroom species cultivated on wheat stalk, Afr. J. Biotechnol. 7(19), 3497-3501 (2008).

Dunkwal, V. y S. Jood, Effect of substrates on nutrient composition of oyster mushroom (Pleurotus sajorcaju), J. Dairying. Foods \& H.S. 28(2), 132-136 (2009).

Frimpong-Manso, J., M. Obodai, M. Dzomeku y M.M. Apertorgbor, Influence of rice husk on biological efficiency and nutrient content of Pleurotus ostreatus (Jacq. Ex. Fr.) Kummer, International Food Research Journal 18, 249-254 (2011).

Ingale, A. y A. Ramteke, Studies on cultivation and biological efficiency of mushrooms grown on different agro-residues, Innovative Romanian Food Biotechnology 6, 25-28 (2010).

Instituto de Nutrición de Centro América y Panamá (INCAP), Tabla de composición de alimentos de Centroamérica, Menchú, M.T. y H. Méndez (ed.), pp 128, Guatemala (INCAP-OPS) (2007).

Jafarpour, M., A. Jalalizand y S. Eghbalsaied, High fiber media as the most efficient substrates for Pleurotus florida culture, Arch. Biol. Sci. Belgrade 63(3), 889-895 (2011). 
Jonathan, S.G., C.B. Oko, A.O. Oyelakiny y O.O. Oluranti, Nutritional values of oyster mushroom (Pleurotus ostreatus) (Jacq. Fr.) Kumm. Cultivated on different agricultural wastes, Nature and Science 10(9), 186-191 (2012).

Martínez-Carrera, D., Oyster mushrooms, McGraw-Hill Yearbook of Science \& Technology 1999, pp. 242245, Licker, D. (ed.), McGraw-Hill, Inc., New York, USA (1998).

Michael, H.W., G. Bultosa y L.M. Pant, Nutritional contents of three edible oyster mushrooms grown on two substrates at Hamramaya, Ethiopia, and sensory properties of boiled mushroom and mushroom sauce, International Journal of Food Science \& Technology 46, 732-738 (2011).

Nyochembeng, L.M., C.A. Beyl y R.P. Pacumbaba, Optimizing edible fungal growth and biodegradation of inedible crop residues using various cropping methods, Bioresource Technology 99, 5645-5649 (2008).

AOAC International, Official Methods of Analysis of AOAC International, Horwitz, W. y G.W. Latimer (ed.), Gaithersbur, Maryland, USA (2010).

Pedneault, K., P. Angers, T.J. Avis, A. Gosseliny y R.J. Tweddell, Fatty acid profiles of polar and non-polar lipids of Pleurotus ostreatus and P. cornucopiae var. "citrino-pileatus" grown at different temperatures, Mycological Research 3, 1228-1234 (2007).

Periasamy, K. y K. Natarajan, Role of lignocellulosic enzymes during basidiomata production by Pleurotus dj amor var. roses, Indian Journal of Biotechnology 3, 577-583 (2004).

Salmones, D., G. Mata y K.N. Waliszewski, Comparative culturing of Pleurotus spp. on coffee pulp and wheat straw: biomass production and substrate biodegradation, Bioresource Technology 96, 537-544 (2005).

Singh, M., B. Vijay, S. Kamal, y G.C. Walkchaure, Mushrooms cultivation, marketing and consumption, pp 278, Directorate of Mushroom Research, Solan, India, (2011).

Stamets, P., Mycelium running: how mushrooms can help save the world, pp 358, Random House Inc, New York, (2005).

Synytsya, A., K. Mícková, I. Jablonský, M. Sluková, y J. Copíkpvá, Mushrooms of genus Pleurotus as a source of dietary fibres and glucans for food supplements, Czech. J. Food Sci. 26(6), 441-446 (2008).

Yildiz, S., U.C. Yildiz, E.D. Gezer y A. Temiz, Some lignocellulosic wastes used as raw material in cultivation of the Pleurotus ostreatus culture mushroom, Process Biochemistry 38, 301-306 (2002).

Zhang, R., X. Li, y J.G. Fadel, Oyster mushroom cultivation whith rice andwheat straw, Biorsource Technology 82, 277-284 (2002).

Zhang, Y., A.E. Ghaly y B. Li, Availability and physical properties of residues from major agricultural crops for energy conversion through thermochemical processes, American Journal of Agricultural and Biological Science 7(3), 312-321 (2012). 
